\title{
NUMERICAL SIMULATION OF THE FLOW OVER A BODY FLYING THROUGH A THERMAL IN A STRATIFIED ATMOSPHERE
}

\author{
I. F. Muzafarov, G. A. Tirskit, S. V. Utyuzhnikov† and N. K. Yamaleev \\ Department of Computational Mathematics, Moscow Institute of Physics and Technology, \\ Dolgoprudny, Moscow 141700, Russia
}

(Received 26 October 1992; in revised form 15 March 1993)

\begin{abstract}
The three-dimensional supersonic viscous flow over a blunted body flying horizontally through a cloud of hot gas (a thermal) is studied numerically. It is assumed that the cloud was formed as the result of a powerful explosion in the Earth's atmosphere. Consideration is given to the late stage of the explosion, corresponding to the vortex ring formation time. A complete problem is unstable. However, the air flow near the body at every point of the flight trajectory can be considered as a quasi-stationary flow, in view of the fact that the ratio of the characteristic time scale in the shock layer to the thermal characteristic time scale is $10^{-5}$. The aerodynamic and heat transfer characteristics of the flying body are studied.
\end{abstract}

\section{THE GOVERNING EQUATIONS}

It is assumed that at the initial moment in time a cloud of hot gas is formed in a stratified Earth atmosphere. The initial thermal parameters are: $R_{\mathrm{T}}=1.2 \mathrm{~km}$, the characteristic thermal lengthscale; and $T_{\max }=16,000 \mathrm{~K}$, the temperature at the explosion center. The temperature distribution in the cloud is represented as

$$
T(z, r)=T_{\mathrm{a}}(z)+\left(T_{\max }-T_{\mathrm{a}}\right) \cdot \exp \left[-\left(\frac{l}{R_{\mathrm{T}}}\right)^{2}\right],
$$

where $T_{\mathrm{a}}(z)$ is the undisturbed Earth atmosphere temperature distribution and $l$ is the distance from the center of the thermal. The maximum temperature location corresponds to the altitude, $H=50 \mathrm{~km}$, measured from the Earth's surface.

The buoyant gas cloud rises, forming an air vortex ring under the action of Archimedes' force which is a maximum at the explosion center. The eddy ring formation is accompanied by intensive turbulent mixing of the hot and cold air layers. To describe the buoyant thermal dynamics the full Navier-Stokes equations with fixed effective viscosity and heat transfer coefficients were used. This turbulence model has been widely used to simulate the buoyant motion of a thermal in a stratified atmosphere [cf. 1-4]. The effective turbulence viscosity coefficient $\mu_{\text {eff }}$ was chosen so that the Reynolds number [calculated from $\mu_{\mathrm{eff}}, R_{\mathrm{T}}, \rho_{\mathrm{T}}$ and $V_{\mathrm{T}} ; \rho_{\mathrm{T}}$ is the undisturbed atmosphere density at the center of the thermal and $V_{\mathrm{T}}=\left(g \cdot R_{\mathrm{T}}\right)^{1 / 2}$ is the characteristic thermal velocity] equals 100 . The effective Prandtl number, $\mathrm{Pr}_{\text {eff }}$, was taken to be 0.72 .

The full unsteady Navier-Stokes equations for the two-dimensional axisymmetric viscous gas flow in the cylindrical coordinate system $\left\{x^{i}\right\}=\{z, r\}$ can be written as

$$
\frac{\partial}{\partial t}(r U)+\frac{\partial}{\partial x^{i}}\left(r E^{i}\right)=F
$$

with:

$$
\begin{gathered}
U=(\rho, \rho u, \rho v, e)^{\mathrm{T}} ; \quad E^{i}=E_{\mathrm{inv}}^{i}-\frac{\mu}{\operatorname{Re}} \cdot E_{\mathrm{vis}}^{i} ; \\
E_{\mathrm{vis}}^{i}=V^{i p}\left(U, U_{x p}\right)+\frac{1}{r} \cdot W^{i}(U)
\end{gathered}
$$

†Author for correspondence. 


$$
\begin{gathered}
E_{\mathrm{inv}}^{1}=\left|\begin{array}{c}
\rho u \\
p+\rho u^{2} \\
\rho u v \\
(e+p) u
\end{array}\right|, \quad E_{\mathrm{inv}}^{2}=\left|\begin{array}{c}
\rho v \\
\rho u v \\
p+\rho v^{2} \\
(e+p) v
\end{array}\right| ; \\
V^{11}=\left|\begin{array}{c}
0 \\
\frac{4}{3} \cdot u_{x^{1}} \\
v_{x^{1}} \\
V^{21}=\left|\begin{array}{c}
1 \\
-\frac{2}{3} \cdot v_{x^{2}} \\
u_{x^{2}} \\
-\frac{2}{3} \cdot u v_{x^{2}}+v u_{x^{2}}
\end{array}\right| \\
0 \\
0 \\
v_{x^{1}} \\
\frac{2}{3} \cdot u_{x^{1}} \\
\frac{2}{3} \cdot v u_{x^{1}}+u v_{x^{1}}+v v_{x^{1}}+\frac{1}{\operatorname{Pr}_{\mathrm{eff}}}
\end{array}\right|, T_{x^{1}}\left|, \quad V^{22}=\right| \begin{array}{c}
u_{x^{2}} \\
\frac{4}{3} \cdot v_{x^{2}} \\
u u_{x^{2}}+\frac{4}{3} v v_{x^{2}}+\frac{1}{\operatorname{Pr}_{\mathrm{eff}}} T_{x^{2}}
\end{array} \mid
\end{gathered}
$$

and

$$
W^{1}=\left|\begin{array}{c}
0 \\
-\frac{2}{3} v \\
0 \\
-\frac{2}{3} u v
\end{array}\right|, W^{2}=\left|\begin{array}{c}
0 \\
0 \\
-\frac{2}{3} v \\
-\frac{2}{3} v^{2}
\end{array}\right|, F=\left|\begin{array}{c}
0 \\
r \rho f^{1} \\
r \rho f^{2}+\left[p+\frac{1}{\operatorname{Re}} \cdot\left(\frac{2}{3} u_{x^{1}}+\frac{2}{3} v_{x^{2}}-\frac{4}{3} \cdot \frac{v}{r}\right)\right] \\
r \rho\left(f^{1} u+f^{2} v\right)
\end{array}\right|,
$$

where $\rho$ is the mass density, $u$ and $v$ are the velocity vector components in the cylindrical coordinate system, $e=0.5 \rho\left(u^{2}+v^{2}\right)+p /(\gamma-1)$ is the specific total energy, $p$ is the pressure and $\gamma=h / e[5]$. Here and henceforth, $f^{i} g_{i}$ denotes the sum

$$
f^{i} g_{i}=\sum_{1}^{2} f^{k} \cdot g_{k}
$$

unless otherwise specified. The length variables are nondimensionalized by the characteristic thermal radius $R_{\mathrm{T}}$. The nondimensionalizations used for the time variable $t$ and for the vector components $f^{\prime}$ and $f^{2}$ are $t^{*} \cdot V_{\mathrm{T}} / R_{\mathrm{T}}$ and $\mathrm{F}^{*} /\left(V_{\mathrm{T}}^{2} / R_{\mathrm{T}}\right)$, respectively, $\operatorname{Re}=\rho_{\mathrm{T}} V_{\mathrm{T}} R_{\mathrm{T}} / \mu_{\mathrm{eff}}$ is the Reynolds number calculated from the thermal parameters.

The viscosity and thermal conductivity are changed by constant values for a flow turbulization influence simulation.

The gas motion has been considered in the rectangle $0 \leqslant r \leqslant r_{1}(t), z_{1}(t) \leqslant z \leqslant z_{2}(t)$. A boundary motion law has been chosen such that near any of the boundaries a gas is considered as close to an undisturbed gas.

The following conditions have been chosen as boundary conditions:

$$
\begin{aligned}
& \text { If } r=0 \quad \text { then } \frac{\partial}{\partial r} f=0, \quad \text { here } f=\{p, \rho, u\} ; \quad v=0 ; \\
& \left.r=r_{1}(t): \frac{\partial u}{\partial r}=0, \quad v=0\right\} p=p_{\text {atm }}, \\
& \left.\left.\begin{array}{l}
z=z_{1}(t): \\
z=z_{2}(t):
\end{array}\right\} \quad u=0, \quad \frac{\partial v}{\partial r}=0\right\} \rho=\rho_{\mathrm{atm}} .
\end{aligned}
$$

These conditions represent the flow symmetry conditions when $r=0$. On the rest of the boundaries the conditions of an undisturbed atmosphere are assumed for the thermodynamic 
functions and the normal velocity component. In addition, extrapolation conditions are used for the tangential velocity component.

Within $15 \mathrm{~s}$ of the beginning of the lift of the thermal, the spherically blunted cone (nose radius $R_{\mathrm{n}}=0.1 \mathrm{~m}$, cone half angle $\theta_{\mathrm{c}}=20^{\circ}$, cone total length $L=2 \mathrm{~m}$ ) flies horizontally into a hot air cloud with velocity $V_{\infty}=2000 \mathrm{~m} / \mathrm{s}$. By the time the body enters the buoyant thermal the gas cloud has the vortex ring form. Therefore, the free stream around the cone is nonhomogeneous and has a three-dimensional structure. It is suggested that the body does not influence the convectivediffusion processes in the thermal. As noted above, the air flow near the cone at every flight trajectory point is treated as a steady flow because the ratio of the characteristic time scale in the shock layer near the body to the thermal time scale is $10^{-5}$. The estimates show that for a turbulent flow in the thermal region the value of Taylor's lengthscale is $10^{3}$ times larger than the shock-layer thickness near the body. As a consequence, there is reason to believe that the flow in the shock layer is laminar.

In flight, the free-stream $\operatorname{Re}$ (calculated with the cone nose radius $R_{\mathrm{n}}$ ) and the free-stream temperature vary from $10^{3}$ to $10^{4}$ and from 200 to $800 \mathrm{~K}$, respectively. Thus, the full viscous shock-layer equations (FVSL) governing the equilibrium chemically reacting flow [6] are used to simulate the flowfield around the body. The FVSL equations used in the present analysis can be obtained from the steady full Navier-Stokes equations by retaining terms up to second order in the square root of the $R e$ in both the viscous and inviscid regions. The set of equations is valid for the region from the body surface to the shock, which is the outer boundary of the flowfield and is found in the course of the solution procedure.

The three-dimensional set of FVSL equations in a body-oriented coordinate system ( $x$-surface coordinate, $y$-normal coordinate, $\varphi$-transverse angle) is written as

$$
\begin{gathered}
\frac{\partial}{\partial x}\left[H_{2} \rho u\right)+\frac{\partial}{\partial \varphi}\left(H_{1} \rho w\right)+\frac{\partial}{\partial y}\left(H_{1} H_{2} \rho v\right)=0 \\
\rho\left[D u-\frac{w^{2}}{H_{1} H_{2}} \frac{\partial H_{2}}{\partial x}+\frac{u w}{H_{1} H_{2}} \frac{\partial H_{1}}{\partial \varphi}+\frac{u v}{H_{1}} \frac{\partial H_{1}}{\partial y}\right]=-\frac{1}{H_{1}} \frac{\partial P}{\partial x}+\frac{1}{H_{1}^{2} H_{2} \operatorname{Re}_{\infty}} \frac{\partial}{\partial y}\left[H_{1}^{3} H_{2} \mu \frac{\partial}{\partial y}\left(\frac{u}{H_{1}}\right)\right], \\
\rho\left[D w+\frac{w u}{H_{1} H_{2}} \frac{\partial H_{2}}{\partial x}-\frac{u^{2}}{H_{1} H_{2}} \frac{\partial H_{1}}{\partial \varphi}+\frac{w v}{H_{2}} \frac{\partial H_{2}}{\partial y}\right]=-\frac{1}{H_{2}} \frac{\partial P}{\partial \varphi}+\frac{1}{H_{1} H_{2}^{2} \operatorname{Re}_{\infty}} \frac{\partial}{\partial y}\left[H_{1} H_{2}^{3} \mu \frac{\partial}{\partial y}\left(\frac{w}{H_{2}}\right)\right], \\
\rho\left[D v-\frac{1}{H_{1}} \frac{\partial H_{1}}{\partial y} u^{2}-\frac{1}{H_{2}} \frac{\partial H_{2}}{\partial y} w^{2}\right]=-\frac{\partial P}{\partial y}
\end{gathered}
$$

and

$$
\rho D H=\frac{1}{H_{1} H_{2}} \frac{\partial}{\partial y}\left\{\frac{H_{2} H_{2} \mu}{\sigma \operatorname{Re}_{\infty}}\left[\frac{\partial H}{\partial y}+\frac{V_{\infty}^{2}(\sigma-1)}{2 H_{\infty}} \frac{\partial\left(u^{2}+w^{2}\right)}{\partial y}-\frac{\sigma u^{2} V_{\infty}^{2}}{H_{\infty} H_{1}} \frac{\partial H_{1}}{\partial y}-\frac{\sigma w^{2} V_{\infty}^{2}}{H_{\infty} H_{2}} \frac{\partial H_{2}}{\partial y}\right]\right\},
$$

with

$$
D=\frac{u}{H_{1}} \frac{\partial}{\partial x}+\frac{w}{H_{2}} \frac{\partial}{\partial \varphi}+v \frac{\partial}{\partial y} ; \quad H=h+\frac{V_{\infty}^{2}}{2 H_{\infty}}\left(u^{2}+w^{2}+v^{2}\right) ; \quad h=\frac{\gamma P V_{\infty}^{2}}{(\gamma-1) \rho H_{\infty}}
$$

where $u, w$ and $v$ are the physical components of the velocity vector in the $x, y$ and $\varphi$ directions, respectively, $\operatorname{Re}_{\infty}=\rho_{\infty} V_{\infty} R_{n} / \mu_{\infty}, \sigma(P, h)$ and $\mu(P, h)$ are the Prandtl number and viscosity coefficient, respectively, for equilibrium chemically-reacting air, $H_{1}=1+y \cdot \aleph$ and $H_{2}=r_{w}+y \cdot \cos \alpha$ are the metric coefficients of the body-oriented coordinate system ( $N$ is the local curvature of the body surface). $R_{\mathrm{n}}$ is the cone nose radius and $\alpha$ is the body surface angle measured from the body axis.

The quantities in equations (2) are nondimensionalized as follows: velocity vector components by $u^{*} / V_{\infty}, w^{*} / V_{\infty}$ and $v^{*} / V_{\infty}$; pressure by $P^{*} /\left(\rho_{\infty} V_{\infty}^{2}\right)$; total enthalpy by $H^{*} / H_{\infty}$. The length quantities are nondimensionalized by the cone nose radius $R_{\mathrm{n}}$. 
In addition to the FVSL equations (2), an equation of state must be specified. The equation of state governing the equilibrium chemically-reacting mixture is

$$
h=\frac{\gamma P V_{\infty}^{2}}{(\gamma-1) \rho H_{\infty}}, \quad \gamma=\gamma(P, h) .
$$

The set of equations (2) is complemented by the following boundary conditions. The RankineHugoniot relations are used at the outer boundary to determine the flow properties immediately behind the shock, which is treated as a discontinuity. These conditions in the body-oriented coordinate system are given as

$$
\begin{aligned}
& v_{\mathrm{s}}=u_{\mathrm{s}} \cdot \tan \beta_{\mathrm{s}}+w_{\mathrm{s}} \cdot \tan \gamma_{\mathrm{s}}+K_{\mathrm{s}} \cdot V_{\infty}(3), \\
& P_{\mathrm{s}}=\frac{1}{\gamma \mathrm{M}_{\infty}^{2}}+\frac{V_{\infty}^{2}(3)\left(1-K_{\mathrm{s}}\right)}{1+\tan ^{2} \beta_{\mathrm{s}}+\tan ^{2} \gamma_{\mathrm{s}}} \\
& u_{\mathrm{s}}=V_{\infty}(1) \cdot \cos ^{2} \beta_{\mathrm{s}}-\frac{w_{\mathrm{s}}}{2} \cdot \sin 2 \beta_{\mathrm{s}} \cdot \tan \gamma_{\mathrm{s}}-\frac{K_{\mathrm{s}}}{2} \cdot V_{\infty}(3) \cdot \sin 2 \beta_{\mathrm{s}}+\frac{\mu_{\mathrm{s}}}{\operatorname{Re}_{\infty} V_{\infty}(3)}\left(\frac{\partial u}{\partial y}-\frac{u}{H_{1}} \frac{\partial H_{1}}{\partial y}\right)_{\mathrm{s}} \\
& w_{\mathrm{s}}=V_{\infty}(2) \cdot \cos ^{2} \gamma_{\mathrm{s}}-\frac{u_{\mathrm{s}}}{2} \cdot \sin 2 \gamma_{\mathrm{s}} \cdot \tan \beta_{\mathrm{s}}-\frac{K_{\mathrm{s}}}{2} \cdot V_{\infty}(3) \cdot \sin 2 \gamma_{\mathrm{s}}+\frac{\mu_{\mathrm{s}}}{\operatorname{Re}_{\infty} V_{\infty}(3)}\left(\frac{\partial w}{\partial y}-\frac{w}{H_{2}} \frac{\partial H_{2}}{\partial y}\right)_{\mathrm{s}}
\end{aligned}
$$

and

$$
H_{\mathrm{s}}=1+\frac{\mu_{\mathrm{s}}}{\sigma \operatorname{Re}_{\infty} V_{\infty}(3)}\left(\frac{\partial H}{\partial y}+\frac{V_{\infty}^{2}(\sigma-1)}{2 H_{\infty}} \frac{\partial\left(u^{2}+w^{2}\right)}{\partial y}-\frac{\sigma u^{2} V_{\infty}^{2}}{H_{\infty} H_{1}} \frac{\partial H_{1}}{\partial y}-\frac{\sigma w^{2} V_{\infty}^{2}}{H_{\infty} H_{2}} \frac{\partial H_{2}}{\partial y}\right),
$$

with

$$
\tan \beta_{\mathrm{s}}=\frac{1}{H_{\mathrm{ls}}} \frac{\partial y}{\partial x} ; \quad \tan \gamma_{\mathrm{s}}=\frac{1}{H_{2 \mathrm{~s}}} \frac{\partial y_{\mathrm{s}}}{\partial \varphi} ; \quad K_{\mathrm{s}}=\frac{1}{\rho_{\mathrm{s}}} .
$$

The subscript "s" denotes that the quantity is taken immediately behind the shock, $V_{\infty}(i), i=\overline{1,3}$, are the free-stream velocity components in the coordinate system $(x, \varphi, y) ; \beta_{\mathrm{s}}$ and $\gamma_{\mathrm{s}}$ are the angles between the bow shock wave and the body surface; $y_{\mathrm{s}}=y_{\mathrm{s}}(x, \varphi)$ is the shock standoff distance.

For the equilibrium chemically-reacting FVSL equations, the boundary conditions at the wall consist of four independent relations representing the nature of the gas mixture and the physical conditions at the wall. These conditions are no slip and no mass transfer conditions:

$$
\left.u\right|_{w}=\left.w\right|_{w}=\left.v\right|_{w}=0,
$$

where the subscript " $w$ " denotes the wall value. The wall temperature $T_{\mathrm{w}}$ is determined as either a fixed value $T_{\mathrm{w}}=500 \mathrm{~K}$ or from the following relation:

$$
q_{\mathrm{w}}=\epsilon^{\prime} \cdot \sigma_{\mathrm{B}} \cdot T_{\mathrm{w}}^{4}
$$

where $q_{\mathrm{w}}$ is the heat transfer, $\epsilon^{\prime}$ is the body surface blackness coefficient and $\sigma_{\mathrm{B}}$ is the Stefan-Boltzmann constant.

The FVSL equations are solved in the region between the body surface and the bow shock wave: $0 \leqslant x \leqslant x_{\mathrm{f}}, 0 \leqslant \varphi \leqslant 2 \pi, 0 \leqslant y \leqslant y_{\mathrm{s}}$. The following conditions are imposed at the surface $x=x_{\mathrm{f}}$ :

$$
\left.\frac{\partial f}{\partial x}\right|_{\mathrm{w}}=0
$$

\section{A NUMERICAL SOLUTION METHOD}

As mentioned above, to simulate the dynamics of a buoyant thermal the two-dimensional full Navier-Stokes equations are solved numerically. 
After a change of variables, $\left\{x^{i}\right\} \rightarrow\left\{\xi^{j}\right\}, i=1,2 ; j=1,2$; the calculation region is a square $[0,1] \times[0,1]:$

$$
\xi^{\prime}=\frac{r}{r_{1}(t)}, \quad \xi^{2}=\frac{z-z_{1}(t)}{z_{2}(t)-z_{1}(t)} .
$$

The governing equations system has been written in the divergency form

$$
\frac{\partial}{\partial t}(\tilde{U})+\frac{\partial}{\partial \xi^{j}}\left(\tilde{E}^{j}\right)=\tilde{F}
$$

with

$$
\tilde{U}=\frac{r}{J} U, \quad \tilde{E}^{j}=\frac{r}{J}\left(\frac{\partial \xi^{j}}{\partial t} U+\frac{\partial \xi^{j}}{\partial x^{i}} E^{i}\right), \quad \tilde{F}=\frac{1}{J} F,
$$

where $J=\operatorname{det}\|\partial \bar{\xi} / \partial \bar{x}\|$, the transformation Jacobian.

Below, the following relations are used (without summaton by $j$ ):

$$
V^{i p}\left(U, U_{x p}\right)=\xi_{x^{k}}^{m} S_{m}^{i k}\left(U, U_{\xi m}\right)
$$

and

$$
\xi_{x^{\prime}}^{j} \xi_{x^{k}}^{j} S_{j}^{i k}\left(U, U_{\xi^{j}}\right)=\xi_{x^{i}}^{j} \xi_{x^{k}}^{j} \frac{\partial}{\partial \xi^{j}} \tilde{S}^{i k}(U) .
$$

In equation (7) the derivatives by $t$ are approximated according to Ref. [7]. The equation system may be written in delta-form:

$$
\left\{I+\frac{\tau \theta_{1}}{1+\theta_{2}} \frac{\partial}{\partial \xi^{j}} Q^{j}\right\} \Delta^{n} \tilde{U}=\text { RHS }
$$

with

$$
Q^{j}=\left\{\xi_{t}^{j} I+\xi_{x^{i}}^{j} \frac{\partial E_{\mathrm{inv}}^{i}}{\partial U}\right\}-\frac{\mu}{\operatorname{Re}} \xi_{x^{i}}^{j}\left\{\xi_{x^{k}}^{j} \frac{r}{J} \frac{\partial}{\partial \xi^{j}} \frac{J}{r} \frac{\partial \widetilde{S}^{i k}}{\partial U}+\frac{1}{r} \frac{\partial W^{i}}{\partial U}\right\}
$$

(without summation by $j$ ) and where

$$
\begin{aligned}
\mathrm{RHS}= & \frac{\tau}{1+\theta_{2}}\left\{\left(I+\theta_{1} \Delta^{n}\right) \tilde{F}-\frac{\partial}{\partial \xi^{j}}\left[\tilde{U}\left(1+\theta_{1} \Delta^{n}\right) \xi_{1}^{j}+\tilde{E}_{\mathrm{inv}}^{i}\left(1+\theta_{1} \Delta^{n}\right) \xi_{x^{i}}^{j}\right]\right. \\
& +\frac{1}{\operatorname{Re}} \frac{\partial}{\partial \xi^{j}}\left[\frac{\partial \tilde{S}^{i k}}{\partial \xi^{j}}\left(1+\theta_{1} \Delta^{n}\right) \mu \frac{r}{J} \xi_{x^{j}}^{j} \xi_{x^{k}}^{j}+\left(I+\theta_{1} \Delta^{n}\right) \mu \frac{r}{J}\right. \\
& \left.\left.\cdot\left(\xi_{x^{i}}^{1} \xi_{x^{k}}^{2} S_{2}^{i k}+\xi_{x^{i}}^{2} \xi_{x^{k}}^{1} S_{1}^{i k}\right)+W^{i}\left(1+\theta_{1} \Delta^{n}\right) \mu \frac{1}{J} \xi_{x^{j}}^{j}\right]\right\}+\frac{\theta_{2}}{1+\theta_{2}} \Delta^{n-1} \tilde{U} .
\end{aligned}
$$

Where $n$ corresponds to a time level number and $\theta_{1}$ and $\theta_{2}$ are parameters,

$$
\frac{\partial}{\partial \xi^{p}}[\cdot] \Delta^{n} U \equiv \frac{\partial}{\partial \xi^{p}}\left([\cdot] \Delta^{n} U\right), \quad \Delta^{n} f=f^{n+1}-f^{n} .
$$

In equation (10) the truncation error by time is $O\left(\left(\theta_{1}-1 / 2-\theta_{2}\right) \times \tau\right)+O\left(\tau^{2}\right)$.

At the right part $F$ was approximated by $\Delta^{n} f \approx \Delta^{n-1} f$ in a calculation of the unknown function $\Delta^{n} f$; in so doing the discretization accuracy was preserved.

In equation (10) all functions are lagged from the $n$th level other than those existing under the operator $\Delta^{n}$.

The equations system (10) is solved with a splitting method in to physics processes and space variables. A one-dimensional boundary problem is solved at each fractional step.

An equation system of the type

$$
\left(I+\frac{\tau \theta_{1}}{1+\theta_{2}} \frac{\partial}{\partial \xi^{p}} Q^{p}\right) \Delta^{n} U=F
$$


(without summation by $p$ ) is approximated by Tolstykh's scheme [8] which has a truncation error $O\left(h^{3}\right)(h$ is the grid step):

$$
\left(B_{P}+\frac{\tau \theta_{1}}{1+\theta_{2}} C_{P} Q^{P}\right) \Delta^{n} U=B_{P} F .
$$

Where $B_{P}$ and $C_{P}$ are the finite-difference operators functioned to the $\xi^{P}$ direction:

$$
B_{P}=A_{0}-1 / 4 \Delta_{0} M
$$

and

$$
C_{P}=1 /(2 h)\left(\Delta_{0}-\Delta_{-}\left(T_{1 / 2} M\right) \Delta_{+}\right) .
$$

The operators $A_{0}, \Delta_{0}, \Delta_{-}, \Delta_{+}$and $T_{1 / 2}$ are running in the following way:

$$
\begin{aligned}
& A_{0} f_{j}=1 / 6\left(f_{j-1}+4 f_{j}+f_{j+1}\right), \\
& \Delta_{0} f_{j}=f_{j+1}-f_{j-1}, \\
& \Delta_{-} f_{j}=f_{j}-f_{j-1}, \quad \Delta_{+} f_{j}=f_{j+1}-f_{j}, \quad T_{1 / 2} f_{j}=f_{j+1 / 2} ;
\end{aligned}
$$

$M=S D S^{-1}$, where $D=\operatorname{sign} \Lambda$ and $\Lambda=\operatorname{diag}\left\{\lambda_{i}\right\}$ matrices, where $\lambda_{i}$ are the latent roots of $Q$. and $S$ is the matrix of the $Q$ latent vectors.

As is known, the Tolstykh scheme has large short wave disturbance numerical dissipation, resulting in attenuation of occurring wear oscillations. Nevertheless, because there are large-amplitude oscillations, doing a calculation is impossible when large graduate flows and flows with strong shock waves are simulated. The following Tolstykh scheme modification has been suggested for overcoming the above-mentioned difficulties. The calculation of each step is carried out by a "predictor-corrector" scheme. The first stage "predictor" corresponds to the basic scheme; in the "corrector" stage the solution $\tilde{U}$ is corrected in the following way [9]:

$$
U_{j}^{n+1}=\tilde{U}_{h}+Q\left(\delta \Phi_{j+1 / 2}-\delta \Phi_{j-1 / 2}\right)
$$

with

$$
\delta \Phi_{j+1 / 2}= \begin{cases}\delta \tilde{U}_{j+1 / 2}, & \text { if }\left(\delta \rho_{j-1 / 2} \delta \rho_{j+1 / 2}<0\right) \text { or }\left(\delta \rho_{j+1 / 2} \delta \rho_{j+3 / 2}<0\right) \\ 0, & \text { in any other case. }\end{cases}
$$

Here $\delta f_{j+1 / 2}=f_{j+1}-f_{j}$.

It takes about $2 \mathrm{~h}$ on an IBM PC AT 386/387 to calculate the rise of the thermal. The grid has $30 \times 50$ nodes.

The equilibrium chemically-reacting air flow in the shock layer near the body is simulated using the three-dimensional FVSL equations. For calculating the required transport coefficients, the tables composed by I. A. Sokolova, S. A. Vasilievskii and A. V. Andriatis are used. The angles of attack during the body flight are small because the thermal velocity is much less than that of the cone. Thus, there is a small parameter in the problem, namely, the angle of attack $\epsilon$. In the flight through the buoyant thermal, the angle of attack undergoes a change at every point of the flight trajectory. The asymptotic method of a small parameter is used to solve the three-dimensional FVSL equations, with the angle of attack as a small parameter. The method has been used previously to solve the three-dimensional Euler and FVSL equations, formerly solved by other authors using the method of time relaxation $[10,11]$. The basic idea of this method is to represent a three-dimensional solution as an asymptotic series expansion with the angle of attack as the expansion parameter. In the present study only the linear terms $O(\epsilon)$ of the series expansion are retained, the higher-order terms are $O\left(\epsilon^{2}\right)[11]$ and may be neglected. The small-parameter method reduces the three-dimensional FVSL equations to two sets of equations: a nonlinear axisymmetric set of equations; and a linear two-dimensional set of equations which is used to determine the first terms of the asymptotic series expansion. The governing system is solved by global iterations with respect to the pressure and the angle of the shock wave. The numerical method for solving the formulated problem is described in detail in Ref. [12]. It takes about $30 \mathrm{~min}$ on an IBM PC 386/387 to calculate one point of the trajectory on the adaptive grid with $80 \times 40$ nodes. 
A simple analysis shows that a small disturbance in the surface pressure distribution may result in significant errors in the pitching moment coefficient in the case of small angles of attack, $\epsilon \rightarrow 0$. Consequently, the calculation of the pitching moment coefficient in the present analysis is based on the small-parameter method [10] because the angle of attack is small.

\section{RESULTS AND DISCUSSION}

Figure 1 shows the velocity vector distribution and the isotherms for the buoyant thermal at the instant the cone enters the thermal. The dashed line corresponds to the body flight trajectory. The time it takes for the cone to fly through the hot gas cloud is about $5 \mathrm{~s}$.

It is easy to show that the time taken to cross a thermal can be approximated by $R_{\mathrm{T}} / V_{\mathrm{T}} \cdot\left[1+O\left(\alpha R_{\mathrm{T}}\right)\right]$, where $R_{\mathrm{T}}$ is the characteristic size of the thermal, $\alpha=\rho^{*} S C_{x}^{*} / M, \rho^{*}$ is the characteristic density, $C_{x}^{*}$ is the characteristic drag coefficient, $S$ is the cross-section of the body and $M$ is its mass. The parameter $\alpha$ is small: for instance, for a body with a length of $2 \mathrm{~m}$, a nose radius of $0.1 \mathrm{~m}$ and a mass of $1000 \mathrm{~kg}, \alpha \approx 10^{-6}-10^{-7} \mathrm{~m}^{-1}$. In the case considered, the flight altitude

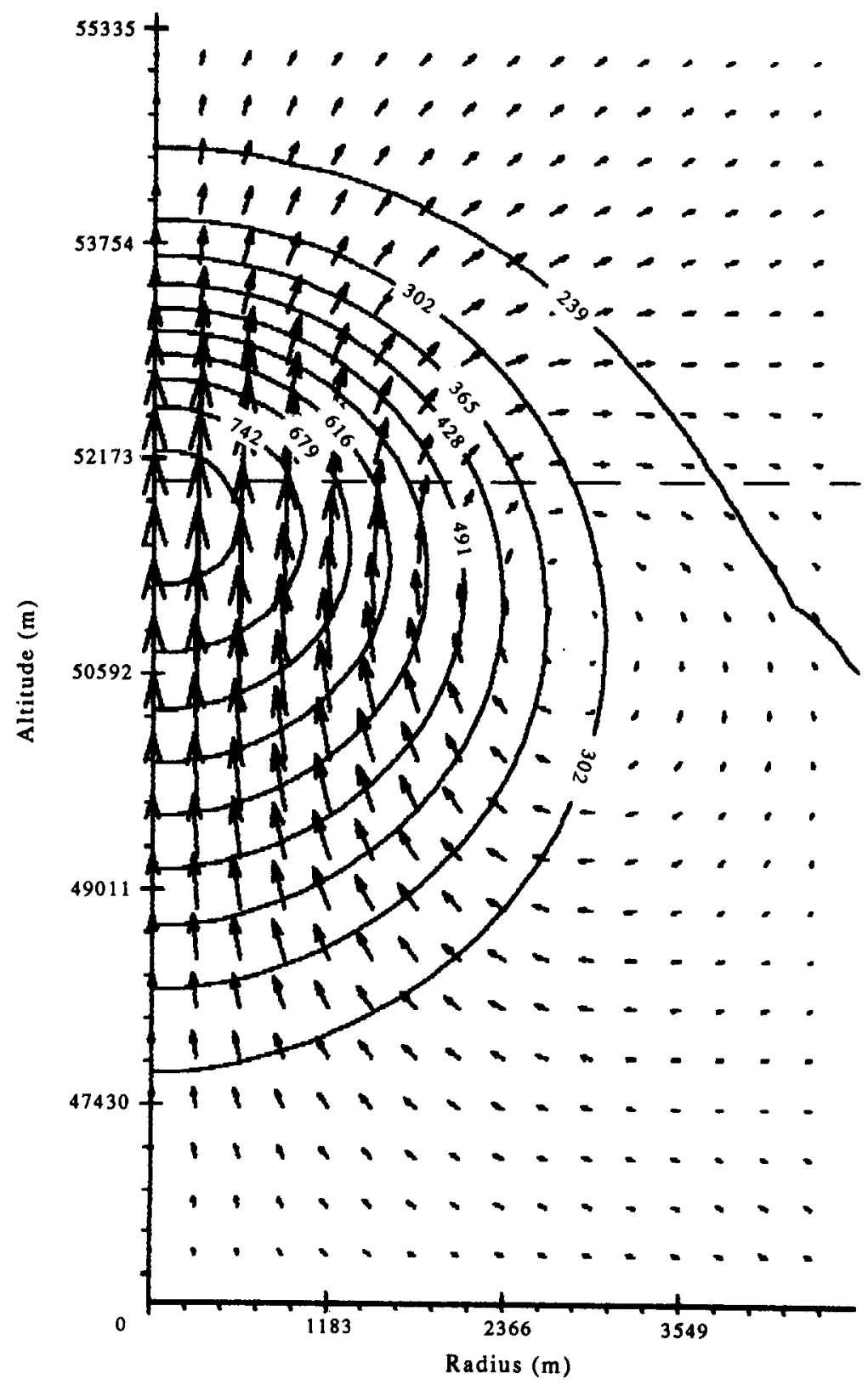

Fig. 1. The velocity field and the temperature isolines in the thermal at time $t_{0}=15 \mathrm{~s}$. 


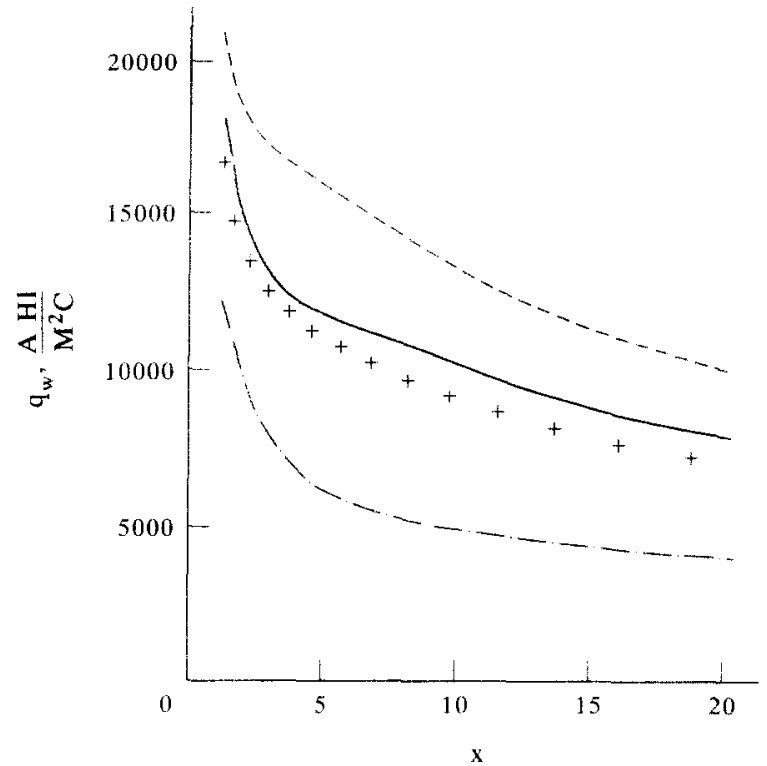

Fig. 2. Distribution of the heat flux for boundary conditions (5).

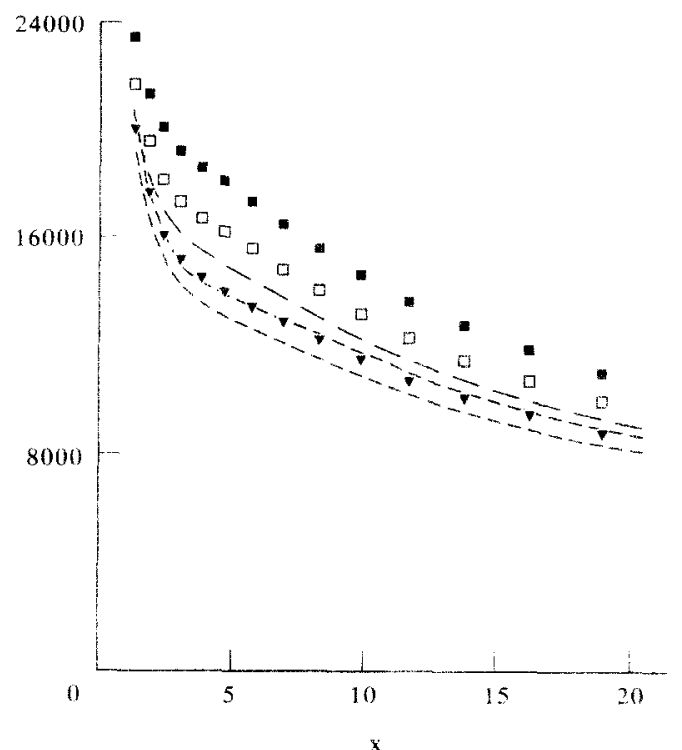

Fig. 3. Distribution of the heat flux. The cooled wall. The windward side

of the body drops by $100 \mathrm{~m}$ at most in a flight time of $5 \mathrm{~s}$. This is $1-2 \%$ of the characteristic size $R_{\mathrm{T}}$. Therefore, the trajectory of the body inside the thermal can be assumed, for simplicity, to be a straight line. Determining more accurately how a thermal influences the flight dynamics requires additional investigations, which are now being carried out.

The heat transfer distributions along the cone surface in the case of boundary condition (5) at the time moments $t_{0}=15.159 \mathrm{~s}$ and $t_{3}=17.802 \mathrm{~s}$ are shown in Fig. 2. Here, the solid line is the heat flux onto the cone at time $t_{0}$, when the body flies out of the thermal. The marked line corresponds to the heat transfer distribution at the time moment $t_{3}$ in that case when the flow around the cone is axisymmetric. It appears that the unexpected drop in the heat transfer in the high-temperature portion of the flight trajectory is due to the fact that the pressure distribution at the same altitude is unchanged in both the thermal and undisturbed atmosphere. Therefore, the cone flies into a rarefied gas cloud, which results in both a drop in the heat transfer and a decrease in the cone surface temperature. The heat flux onto the windward side at time $t_{3}(-\ldots)$ is greater than that at time $t_{0}$ because the angle of attack at the trajectory point is not zero and equals $\epsilon=6.83$. The - - line corresponds to the heat transfer on the leeward side of the cone.

In Figs 3-6 the results obtained for six cone flight trajectory points for $T_{w}=500 \mathrm{~K}$ are given. The free-stream conditions, angle of attack and line marker at the corresponding flight trajectory point are specified in Table 1 . The free-stream conditions at the entry point into the thermal completely coincide with the free-stream conditions at the instant the cone leaves the thermal. Therefore, the line markers corresponding to these flight trajectory points are not distinguished in Figs 3-6.

Figures 3 and 4 show the heat transfer coefficients along the cone surface for the windward and leeward sides, respectively. The leeward side heat transfer distribution at every trajectory point is less than the heat transfer at the initial trajectory point, where the flow around the cone is

Table 1

\begin{tabular}{ccccccc}
\hline Time (s) & $T_{x}(\mathrm{~K})$ & $\mathrm{Re}_{x} \cdot 10^{-3}$ & $\mathbf{M}_{x}$ & $\rho_{x} \cdot 10^{2}\left(\frac{\mathbf{k g}}{\mathbf{m}^{3}}\right)$ & $\epsilon(\mathrm{deg})$ & Line/symbol \\
\hline$t_{0}=15.159$ & 232.2 & 13.4 & 6.55 & 0.101 & 0 & \\
$t_{1}=16.591$ & 592.8 & 9.72 & 4.83 & 0.0544 & 1.17 & $\nabla$ \\
$t_{2}=16.832$ & 808.8 & 5.95 & 4.31 & 0.0436 & 2.65 & - \\
$t_{3}=17.802$ & 794.6 & 6.28 & 3.69 & 0.0329 & 6.83 & $\square$ \\
$t_{4}=18.299$ & 703.6 & 7.59 & 3.99 & 0.0376 & 4.54 & $\square$ \\
$t_{3}=19.044$ & 536.9 & 11.6 & 5.45 & 0.0689 & 0.26 & - \\
$t_{6}=20.159$ & 219.2 & 55.0 & 6.55 & 0.101 & 0 & $\ldots$ \\
\hline
\end{tabular}




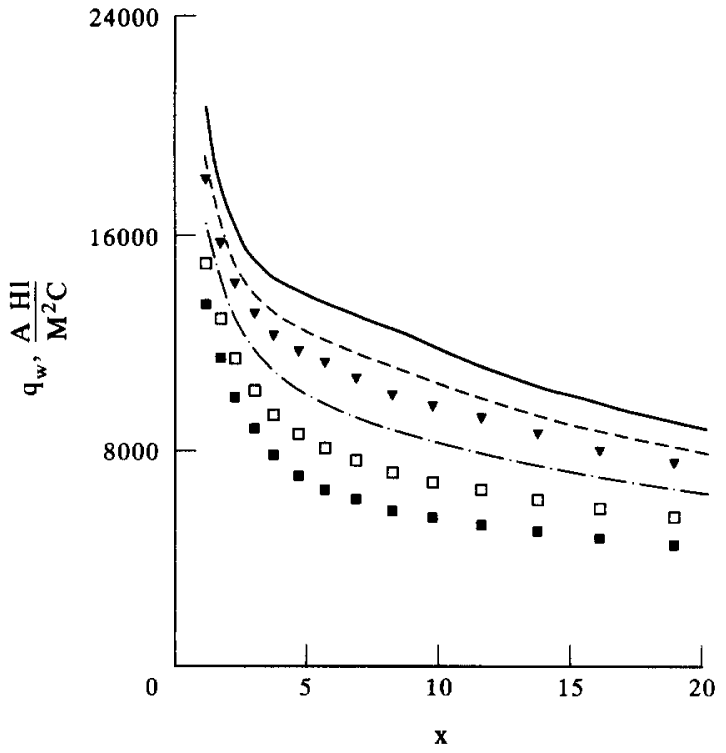

Fig. 4. Distribution of the heat flux. The cooled wall. The leeward side.

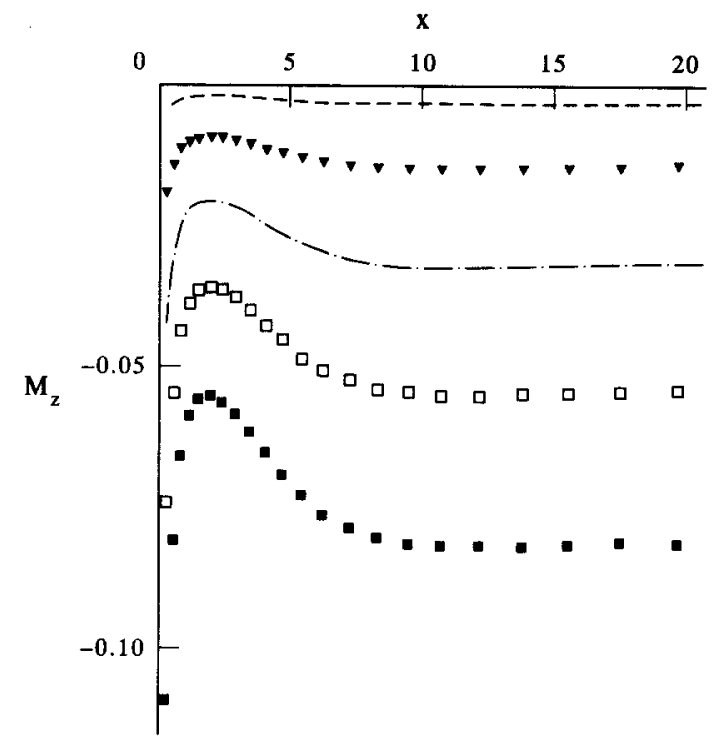

Fig. 5. The pitching moment.

axisymmetric. The heat transfer time-dependence during the cone flight through the thermal changes nonmonotonically along the flight trajectory. This is explained by the variation in the angle of attack. The variation in the windward side heat transfer during the flight is governed by two factors: the first is the increase in the angle of attack; and the second is the decrease in the free-stream air density. The two factors act in opposite directions. As a result, the change in the windward side heat transfer distribution is less than that on the leeward side.

The pitching moment coefficient distributions for six flight trajectory points are shown in Fig. 5. The pitching moment coefficient is given by

$$
M_{z}(z)=\frac{2}{\rho_{\infty} U_{\infty}^{2} S z} \cdot \int_{\Sigma} \mathbf{r} \times \mathbf{P}_{n} \mathrm{~d} s .
$$

Here $\mathbf{P}_{n}$ is the stress vector, $\Sigma$ is the cone surface and $S(z)$ is the cross-section area in $z=$ const.

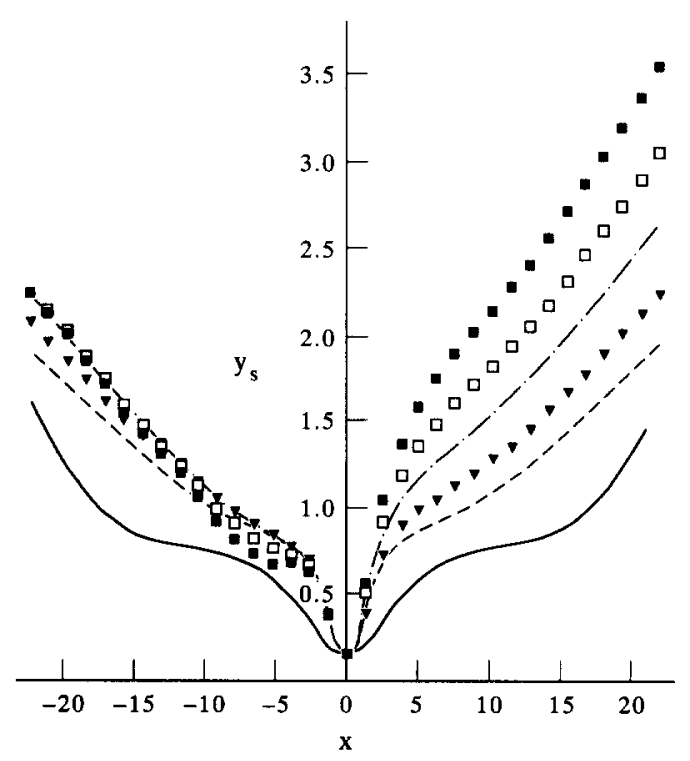

Fig. 6. The shock standoff distance. 
Figure 6 shows the shock standoff distance in both the windward and leeward cone sides for six flight trajectory points. In Fig. 6 the $x$ coordinate increases in the leeward side direction and the point $x=0$ corresponds to the cone nose tip.

\section{CONCLUSION}

The problem of the flight of a blunted body across a large-scale thermal in a stratified atmosphere is formulated. A numerical algorithm is constructed to solve the problem. The Navier-Stokes equations of the gas motion in a thermal are integrated using a compact finite-difference scheme. The problem of the flow past the body is solved using the asymptotic method of a small parameter and the method of global iterations. Distributions of the heat flux on the body in space and time, the standoff distance of the shock wave and the pitching moment are calculated. The heat flux on the body is shown to be, in general, smaller in a thermal. The heat flux on the windward surface of the body is not a monotonic function of time.

\section{REFERENCES}

1. U. A. Gostintsev, A. F. Solodovnik, V. V. Lazarev and U. V. Shatskikh, Turbulent thermal in a stratified atmosphere. Proc. Inst. Chemical Physics, Chernogolovka, p. 46 (1975) (in Russian).

2. U. A. Gostintsev and L. A. Sukhanov, Turbulent thermal of large viscosity in nonstratified atmosphere. Proc. U.S.S.R. Acad. Sci. Fluid Gas Mechanics 15, 153 (1984) (in Russian).

3. V. A. Andruschenko, A. A. Gorbunov, H. S. Kestenboym and L. A. Chudov, Numerical simulation of the three-dimensional interaction of large scale thermals. Proc. U.S.S.R. Acad. Sci. Fluid Gas Mechanics 21, 53 (1990) (in Russian).

4. N. A. Kudryashov and V. M. Prostockishin, The influence of altitude on the thermal dynamics in homogeneous atmosphere. Proc. U.S.S.R. Acad. Sci. Fluid and Gas Mechanics 21, 582 (1990) (in Russian).

5. V. V. Lunev, Hypersonic Aerodynamics. Mashinostroenie, Moscow (1975) (in Russian).

6. A. A. Gershbein, S. V. Peigin and G. A. Tirskii, The calculation of supersonic flow past a body at small and moderate Reynolds numbers. In Advances in Science and Technology of VINITI. Mechanics of Fluids and Gases Series, Vol. 19, pp. 3-85 (1985) (in Russian).

7. R. M. Beam and R. F. Warming, An implicit factored scheme for the compressible Navier-Stokes equations. AlAA $J l 16,393$ (1978).

8. A. I. Tolstykh, Compact Difference Schemes and Their Applications to Fluid Dynamics Problems. Nauka, Moscow (1990) (in Russian).

9. A. I. Zhmakin and A. A. Fursenko, About one monotonic difference shock-capturing scheme. J. Comput. Math. Math. Phys. 20, 1021 (1980) (in Russian).

10. V. I. Livinsky, Application of the small parameter method to the supersonic flow problems. Preprint No. 167, Inst. Appl. Math., U.S.S.R. Academy of Sciences, Moscow (1984) (in Russian).

11. V. E. Karyakin, Numerical simulation of supersonic viscous flow over axisymmetric body at small angle of attack. J. Comput. Math. Math. Phys. 21, 150 (1981) (in Russian).

12. G. A. Tirskii, S. V. Utyuzhnikov and N. K. Yamaleev, Efficient numerical method for simulation of supersonic viscous flow past a blunted body at small angle of attack. Computers Fluids 23, 103 (1994). 